Supporting Information for:

\title{
A New Approach to Natural Products Chemistry Exemplified by the Identification of Sulfated Nucleosides in Spider Venom
}

\author{
Andrew E. Taggi, Jerrold Meinwald, and Frank C. Schroeder* \\ The Cornell Institute for Research in Chemical Ecology and The Department of Chemistry and \\ Chemical Biology, Baker Laboratory, Cornell University, Ithaca, New York, 14853. \\ E-mail:fs31@cornell.edu
}

General. All solvents used were commercially available from Aldrich and Acros Chemicals. Tegenaria agrestis were collected, identified, reared, and milked by Fauna Laboratories, Ltd (Almaty, Kazakhstan). The venom was acquired via electric stimulation ${ }^{1}$ of the venom gland and then immediately lyophilized and stored at $-20^{\circ} \mathrm{C}$. NMR spectra were recorded at $25^{\circ} \mathrm{C}$ using aVarian INOVA 500 (500 MHz proton, $126 \mathrm{MHz}$ carbon) spectrometer with $\mathrm{D}_{2} \mathrm{O}$ as the solvent. The ${ }^{1} \mathrm{H}$ and ${ }^{13} \mathrm{C}$ chemical shifts are given in parts per million $(\delta)$ with respect to residual solvent peaks, while coupling constants (J) are given in Hz. Double quantum filtered COSY (dqf-COSY) spectra were acquired using the standard Varian pulse sequence and phase cycling. Phasesensitive NOESY spectra were acquired with a mixing time of $500 \mathrm{~ms}$. HMQC spectra were acquired without decoupling in the phase-sensitive, mode without gradients, using phase-cycling for coherence selection. Similarly, magnitude-mode HMBC spectra were also acquired without gradients and using phase-cycling for coherence selection. Spectra for 2, 3, 7, and $\mathbf{8}$ were acquired using Shigemi NMR Microtubes $\left(\mathrm{D}_{2} \mathrm{O}\right)$. Mass spectrometry was carried out on a Micromass Quattro II (negative ion electrospray ionization). HPLC fractionation was carried out on an Agilent 1100 series HPLC system with diode-array detector (190-900 nm) using a Supleco Discovery HS C-18 column ( $25 \mathrm{~cm} \times 10 \mathrm{~mm}, 5$ _m particle diameter) with a $0.025 \%$ TFA in water/methanol gradient and a solvent flow rate of $3.4 \mathrm{~mL} / \mathrm{min}$. Natural product 1 (HF-7) was previously characterized. ${ }^{2}$

\begin{tabular}{|c|c|}
\hline \multicolumn{2}{|c|}{ HPLC Method } \\
\hline Time & \% $\mathbf{C H}_{3} \mathbf{O H}$ in TFA/Water \\
\hline 0 & 5 \\
\hline 3 & 5 \\
\hline 10 & 25 \\
\hline 12 & 100 \\
\hline 17 & 100 \\
\hline 18 & 5 \\
\hline 25 & Stop \\
\hline
\end{tabular}

1 (a) Grant, J. B.; Land, B. Herpetological Review, 2002, 33, 38-41. (b) Norment, B. R.; Smith, O. E.; Toxicon, 1968, 7, 141-144.

${ }^{2}$ McCormick, J.; Li, Y.; McCormick, K.; Duynstee, H. I.; van Engen, A. K.; van der Marel, G. A.; Ganem, B.; van Boom, J. H.; Meinwald, J. J. Am. Chem. Soc. 1999, 121, 5661-5665. 
General Procedure for the Analysis of the Venom of Tegenaria agrestis. Venom $(235 \mu \mathrm{L})$ was obtained in lyophilized form (white powder, $31 \mathrm{mg}$ ). To this material was added $0.75 \mathrm{~mL}$ of $\mathrm{D}_{2} \mathrm{O}(100$ atom $\% \mathrm{~d})$. The resulting suspension was subjected to sonication for 1 minute and then centrifuged for 5 minutes. Subsequently, ${ }^{1} \mathrm{H}$ NMR, dfq-COSY, and HMBC spectra of the supernatant were acquired. After acquisition of the NMR spectra, the sample was purified by HPLC, collecting three fractions: early $(\mathbf{A}, \mathrm{t}=0 \rightarrow 9 \mathrm{~min})$, middle $(\mathbf{B}, \mathrm{t}=9 \rightarrow 15 \mathrm{~min})$ and late $(\mathbf{C}, \mathrm{t}=15 \rightarrow 25 \mathrm{~min})$. Appropriate amounts of $\mathrm{d}_{5}$-pyridine were immediately added to each fraction. Each fraction was concentrated on a rotary evaporator at ambient temperature and then reexamined by ${ }^{1} \mathrm{H}$ NMR and dfq-COSY. Fractions $\mathbf{A}$ and $\mathbf{B}$ contained nucleosides $\mathbf{1}-\mathbf{8}$, while fraction $\mathbf{C}$ contained acylpolyamines, small peptides, and proteins. Fractions $\mathbf{A}$ and $\mathbf{B}$ were further purified by HPLC, this time collecting all of the major peaks separately. Once again, each fraction was quenched with $d_{5}$-pyridine prior to concentration. Natural products $\mathbf{1}-\mathbf{8}$ were identified using ${ }^{1} \mathrm{H}$ NMR, dfq-COSY, HMBC, HMQC, and NOESY experiments. Mass spectra were acquired via ESI-MS (30\% $\mathrm{CH}_{3} \mathrm{OH} /$ water) in the negative ion mode.
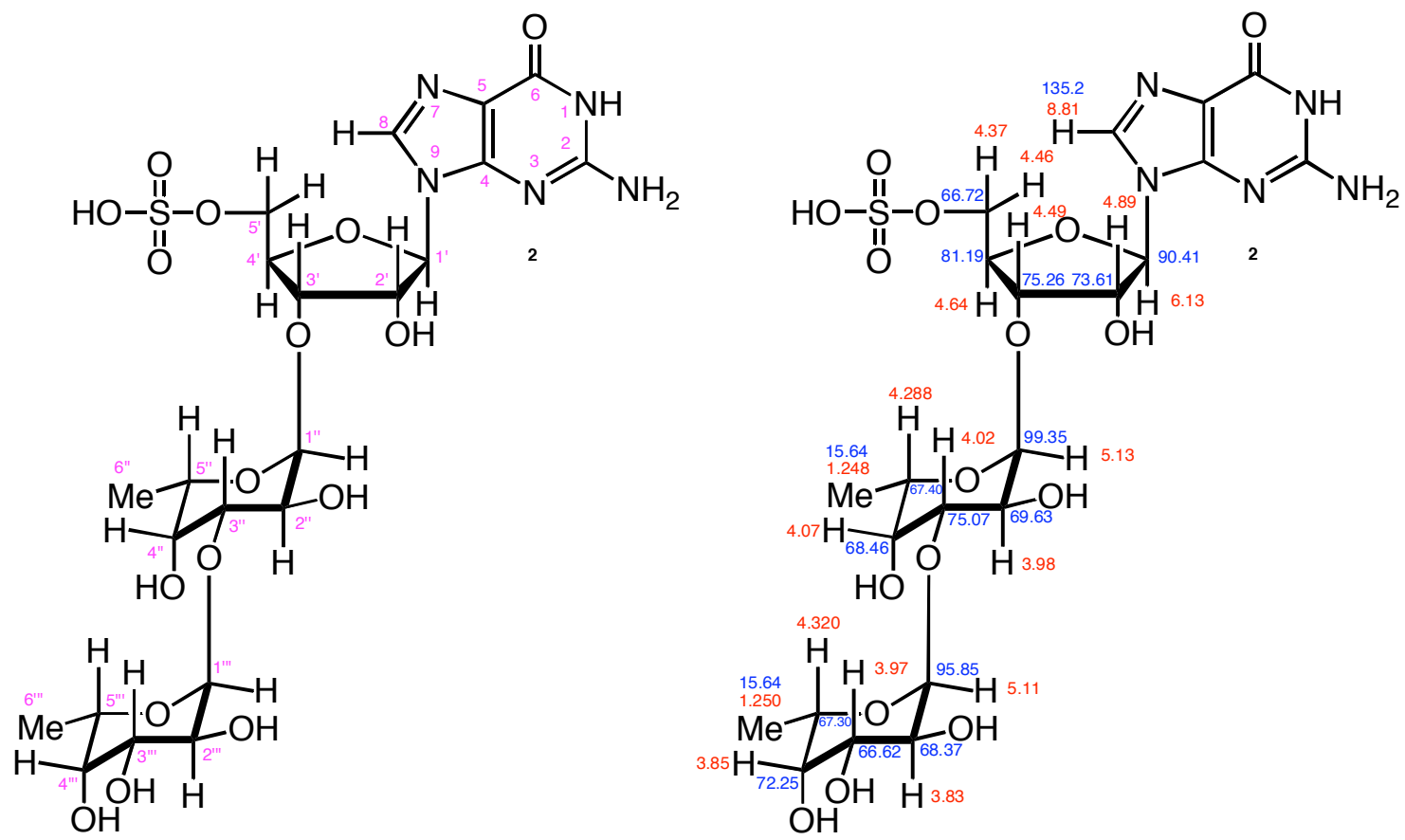

((2R,3R,4S,5R)-5-(2-amino-6-oxo-1,6-dihydropurin-9-yl)-3-(2S,4S,5R)-3,5-dihydroxy-6methyl-4-(2S,4S,5S)-3,4,5-trihydroxy-6-methyl-tetrahydro-2H-pyran-2-yloxy)-tetrahydro2H-pyran-2-yloxy)-4-hydroxy-tetrahydrofuran-2-yl)methyl hydrogen sulfate) (2). HPLC $\left(\mathrm{CH}_{3} \mathrm{OH} / 0.025 \%\right.$ TFA in water, $\left.3.4 \mathrm{~mL} / \mathrm{min}\right) \mathrm{t}=10.5 \mathrm{~min} . \mathrm{UV}_{\max }=252,278 \mathrm{~nm}$. ESI-MS (negative ion): $(\mathrm{M}-1) / \mathrm{Z}=654.16 \mathrm{AMU}$.

\begin{tabular}{|c|l|l|l|l|}
\hline Position & \multicolumn{1}{|c|}{$\boldsymbol{\delta}^{13} \mathbf{C}[\mathbf{p p m}]$} & $\boldsymbol{\delta}^{1} \mathbf{H}[\mathbf{p p m}]$ & $\begin{array}{c}\text { Relevant } \\
\text { HMBC } \\
\text { Correlations }\end{array}$ & $\begin{array}{c}\text { Relevant NOESY } \\
\text { Correlations }\end{array}$ \\
\hline 8 & $135.2\left(\mathrm{~J}_{\mathrm{C}-\mathrm{H}}=215.9\right)$ & $8.81(\mathrm{bs})$ & $\mathrm{C} 1{ }^{\prime}$ & \\
\hline $1^{\prime}$ & $90.41\left(\mathrm{~J}_{\mathrm{C}-\mathrm{H}}=177.3\right)$ & $6.13\left(\mathrm{~J}_{1^{\prime}-2},=3.7\right)$ & $\mathrm{C} 8$ & \\
\hline
\end{tabular}




\begin{tabular}{|c|c|c|c|c|}
\hline 2' & $73.61\left(\mathrm{~J}_{\mathrm{C}-\mathrm{H}}=157.6\right)$ & $4.89\left(\mathrm{~J}_{2^{\prime}-3^{\prime}}=5.3\right)$ & & H 3' \\
\hline 3 ' & $75.26\left(\mathrm{~J}_{\mathrm{C}-\mathrm{H}}=152.5\right)$ & $4.49\left(\mathrm{~J}_{3^{\prime}-4},=6.0\right)$ & C 1", & H 2', H 1', \\
\hline $4{ }^{\prime}$ & $81.19\left(\mathrm{~J}_{\mathrm{C}-\mathrm{H}}=150.4\right)$ & $\begin{aligned} 4.64\left(\mathrm{~J}_{4^{\prime}-5{ }^{\prime} \mathrm{a}}=3.3\right) \\
\left(\mathrm{J}_{4^{\prime}-5^{\prime} \mathrm{b}}=2.7\right)\end{aligned}$ & & H 1', \\
\hline $\begin{array}{l}5 \text { 'a } \\
5 \text { 'b }\end{array}$ & $66.72\left(\mathrm{~J}_{\mathrm{C}-\mathrm{H}}=144.2\right)$ & 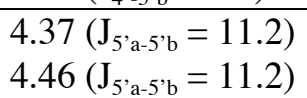 & & \\
\hline $1 \%$ & $99.35\left(\mathrm{~J}_{\mathrm{C}-\mathrm{H}}=176.3\right)$ & $5.13\left(\mathrm{~J}_{1,-2,}=4.0\right)$ & C 3', C 5', & H 3', H4' \\
\hline 2 ' & $69.63\left(\mathrm{~J}_{\mathrm{C}-\mathrm{H}}=144.2\right)$ & $3.98\left(\mathrm{~J}_{2^{\prime \prime}-3^{\prime \prime}}=10.5\right)$ & & \\
\hline $3 "$ & $75.07\left(\mathrm{~J}_{\mathrm{C}-\mathrm{H}}=146.2\right)$ & $4.02\left(\mathrm{~J}_{3^{\prime \prime}-4,}=3.3\right)$ & C 1',' & H 5', H1', \\
\hline $4 "$ & $68.46\left(\mathrm{~J}_{\mathrm{C}-\mathrm{H}}=148.3\right)$ & $4.07\left(\mathrm{~J}_{4},{ }^{\prime}-5,<1\right)$ & & H 1,", \\
\hline 5, & $67.40\left(\mathrm{~J}_{\mathrm{C}-\mathrm{H}}=149.4\right)$ & $4.29\left(\mathrm{~J}_{5,-}, 6^{\prime},=6.6\right)$ & C 1", & H 3,' \\
\hline $6 "$ & $15.64\left(\mathrm{~J}_{\mathrm{C}-\mathrm{H}}=126.5\right)$ & 1.248 & & \\
\hline $1, "$, & $95.85\left(\mathrm{~J}_{\mathrm{C}-\mathrm{H}}=173.2\right)$ & $5.11\left(\mathrm{~J}_{1},{ }^{\prime}, 2, ",=4.0\right)$ & C 3', C 5', & H 3', H 4', \\
\hline $2, \%$ & $68.37\left(\mathrm{~J}_{\mathrm{C}-\mathrm{H}}=146.2\right)$ & $3.83\left(\mathrm{~J}_{2, \cdots},{ }^{\prime},,=10.5\right)$ & & \\
\hline $3^{\prime \prime \prime}$ & $66.62\left(\mathrm{~J}_{\mathrm{C}-\mathrm{H}}=147.3\right)$ & $3.97\left(\mathrm{~J}_{3^{\prime \prime \prime}-4^{\prime \prime},}=3.3\right)$ & & H 5,', \\
\hline $4^{\prime \prime \prime}$ & $72.25\left(\mathrm{~J}_{\mathrm{C}-\mathrm{H}}=147.3\right)$ & $3.85\left(\mathrm{~J}_{4, ",-5,,}<1\right)$ & & \\
\hline $5^{\prime, \prime}$ & $67.30\left(\mathrm{~J}_{\mathrm{C}-\mathrm{H}}=152.5\right)$ & $4.32\left(\mathrm{~J}_{5},{ }_{-6},{ }^{\prime},=6.6\right)$ & C 1', & H 3,", \\
\hline $6, \%$ & $15.64\left(\mathrm{~J}_{\mathrm{C}-\mathrm{H}}=126.5\right)$ & 1.250 & & \\
\hline
\end{tabular}

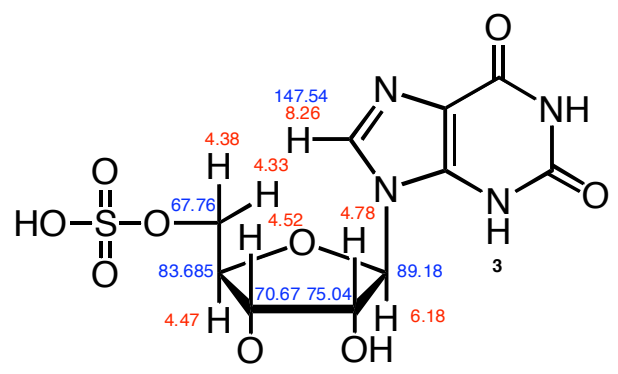

((2R,3R,4S,5R)-5-(2,6-dioxo-1,2,3,6-tetrahydropurin-9-yl)-3,4-dihydroxy-tetrahydrofuran-2yl)methyl hydrogen sulfate (3). HPLC $\left(\mathrm{CH}_{3} \mathrm{OH} / 0.025 \%\right.$ TFA in water, $\left.3.4 \mathrm{~mL} / \mathrm{min}\right) \mathrm{t}=7.2$ min. $\mathrm{UV}_{\max }=249 \mathrm{~nm}$. ESI-MS (negative ion): $(\mathrm{M}-1) / \mathrm{Z}=363.02 \mathrm{AMU}$.

\begin{tabular}{|c|c|c|c|}
\hline Position & $\delta^{13} \mathrm{C}$ [ppm] & $\delta^{1} \mathbf{H}[\mathrm{ppm}]$ & $\begin{array}{c}\text { Relevant HMBC } \\
\text { Correlations }\end{array}$ \\
\hline 8 & $147.5\left(\mathrm{~J}_{\mathrm{C}-\mathrm{H}}=212.3\right)$ & $8.26(\mathrm{bs})$ & C 1' \\
\hline 1 ' & $89.18\left(\mathrm{~J}_{\mathrm{C}-\mathrm{H}}=169.4\right)$ & $6.18\left(\mathrm{~J}_{1^{\prime}-2},=4.9\right)$ & C 8 \\
\hline 2 ' & $75.04\left(\mathrm{~J}_{\mathrm{C}-\mathrm{H}}=152.0\right)$ & $4.78\left(\mathrm{~J}_{2^{\prime}-3},=5.4\right)$ & \\
\hline $3{ }^{\prime}$ & $70.67\left(\mathrm{~J}_{\mathrm{C}-\mathrm{H}}=152.7\right)$ & $4.52\left(\mathrm{~J}_{3^{\prime}-4},=4.4\right)$ & \\
\hline $4^{\prime}$ & $83.69\left(\mathrm{~J}_{\mathrm{C}-\mathrm{H}}=152.5\right)$ & $\begin{aligned} 4.47\left(\mathrm{~J}_{4^{\prime}-5^{\prime} \mathrm{a}}=3.6\right) \\
\left(\mathrm{J}_{4^{\prime}-5^{\prime} \mathrm{b}}=2.8\right)\end{aligned}$ & \\
\hline $\begin{array}{l}5 ' \mathrm{a} \\
5{ }^{\prime} \mathrm{b}\end{array}$ & $67.76\left(\mathrm{~J}_{\mathrm{C}-\mathrm{H}}=151.0\right)$ & 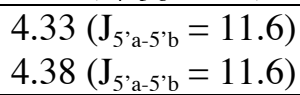 & \\
\hline
\end{tabular}




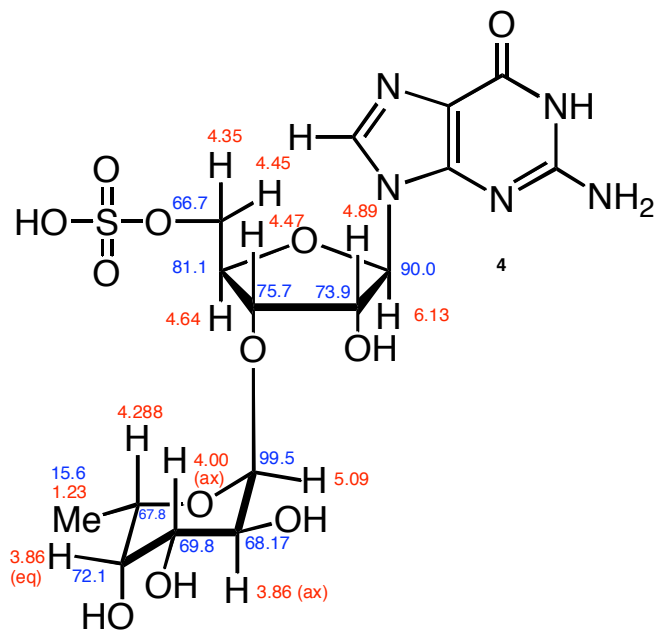

$((2 R, 3 R, 4 S, 5 R)-5-(2-a m i n o-6-0 x o-1,6-d i h y d r o p u r i n-9-y l)-4-h y d r o x y-3-((2 S, 4 S, 5 S)-3,4,5-$ trihydroxy-6-methyl-tetrahydro-2H-pyran-2-yloxy)-tetrahydrofuran-2-yl)methyl hydrogen sulfate (4). $\mathrm{HPLC}\left(\mathrm{CH}_{3} \mathrm{OH} / 0.025 \%\right.$ TFA in water, $\left.3.4 \mathrm{~mL} / \mathrm{min}\right) \mathrm{t}=9.1 \mathrm{~min} . \mathrm{UV}_{\max }=252,278$ nm. ESI-MS (negative ion): (M-1)/Z $=508.10$ AMU.

\begin{tabular}{|c|c|c|c|c|}
\hline Position & $\delta{ }^{13} \mathrm{C}[\mathrm{ppm}]$ & $\delta^{1} \mathbf{H}[\mathrm{ppm}]$ & $\begin{array}{c}\text { Relevant } \\
\text { HMBC } \\
\text { Correlations }\end{array}$ & $\begin{array}{c}\text { Relevant NOESY } \\
\text { Correlations }\end{array}$ \\
\hline 8 & $135.5\left(\mathrm{~J}_{\mathrm{C}-\mathrm{H}}=216.5\right)$ & 8.80 (bs) & C 1' & \\
\hline $1^{\prime}$ & $90.0\left(\mathrm{~J}_{\mathrm{C}-\mathrm{H}}=174.7\right)$ & $6.13\left(\mathrm{~J}_{1^{\prime}-2^{\prime}}=3.6\right)$ & $\mathrm{C} 8$ & \\
\hline 2 ' & $73.9\left(\mathrm{~J}_{\mathrm{C}-\mathrm{H}}=155.5\right)$ & $4.89\left(\mathrm{~J}_{2^{\prime}-3^{\prime}}=5.1\right)$ & & \\
\hline 3 & $75.7\left(\mathrm{~J}_{\mathrm{C}-\mathrm{H}}=151.4\right)$ & $4.47\left(\mathrm{~J}_{3^{3}-4^{\prime}}=6.0\right)$ & C 1", & H 1", \\
\hline 4 ' & $81.1\left(\mathrm{~J}_{\mathrm{C}-\mathrm{H}}=151.4\right)$ & $\begin{aligned} 4.64\left(\mathrm{~J}_{4^{\prime}-5^{\prime} \mathrm{a}}\right. & =3.1) \\
\left(\mathrm{J}_{4^{\prime}-5^{\prime} \mathrm{b}}\right. & =2.6)\end{aligned}$ & & H 1", \\
\hline $\begin{array}{l}5 ' \mathrm{a} \\
5{ }^{\prime} \mathrm{b}\end{array}$ & $\begin{aligned} 66.7\left(\mathrm{~J}_{\mathrm{C}-\mathrm{H}}\right. & =145.5) \\
\left(\mathrm{J}_{\mathrm{C}-\mathrm{H}}\right. & =145.5)\end{aligned}$ & 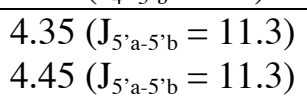 & & \\
\hline 1, & $99.5\left(\mathrm{~J}_{\mathrm{C}-\mathrm{H}}=173.2\right)$ & $5.09\left(\mathrm{~J}_{1,-2,}=4.0\right)$ & C 3', C 5', & H 3', H4' \\
\hline $2 \%$ & $68.2\left(\mathrm{~J}_{\mathrm{C}-\mathrm{H}}=142.4\right)$ & $3.86\left(\mathrm{~J}_{2^{\prime \prime}-3^{\prime \prime}}=10.4\right)$ & & \\
\hline 3, & $69.8\left(\mathrm{~J}_{\mathrm{C}-\mathrm{H}}=144.3\right)$ & $4.00\left(\mathrm{~J}_{3^{\prime \prime}-4,}=3.3\right)$ & & $\mathrm{H} \mathrm{5}$ \\
\hline $4 "$ & $72.1\left(\mathrm{~J}_{\mathrm{C}-\mathrm{H}}=146.2\right)$ & $3.86\left(\mathrm{~J}_{4}{ }^{\prime},-5,<1\right)$ & & \\
\hline 5, & $67.8\left(\mathrm{~J}_{\mathrm{C}-\mathrm{H}}=147.7\right)$ & $4.29\left(\mathrm{~J}_{5,-}, 6^{,},=6.6\right)$ & C 1"' & H 3', \\
\hline $6 "$ & $15.6\left(\mathrm{~J}_{\mathrm{C}-\mathrm{H}}=128.6\right)$ & 1.23 & & \\
\hline
\end{tabular}

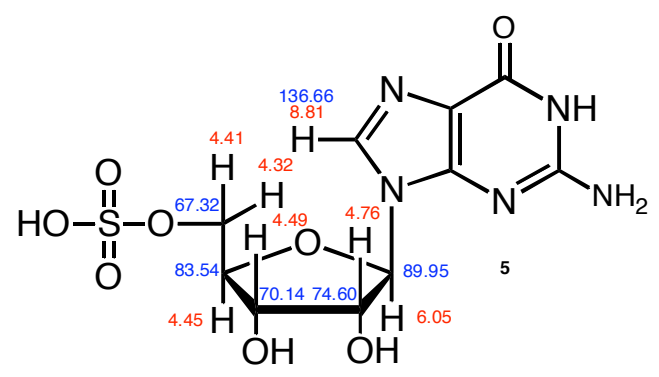

$((2 R, 3 R, 4 S, 5 R)-5$-(2-amino-6-oxo-1,6-dihydropurin-9-yl)-3,4-dihydroxy-tetrahydrofuran-2-

yl)methyl hydrogen sulfate (5). $\mathrm{HPLC}\left(\mathrm{CH}_{3} \mathrm{OH} / 0.025 \% \mathrm{TFA}\right.$ in water, $\left.3.4 \mathrm{~mL} / \mathrm{min}\right) \mathrm{t}=8.5$ min. $\mathrm{UV}_{\max }=254,280 \mathrm{~nm}$. ESI-MS (negative ion): $(\mathrm{M}-1) / \mathrm{Z}=362.04$ AMU. 


\begin{tabular}{|c|c|c|c|}
\hline Position & $\delta^{13} \mathrm{C}[\mathrm{ppm}]$ & $\delta^{1} \mathbf{H}[\mathrm{ppm}]$ & $\begin{array}{c}\text { Relevant HMBC } \\
\text { Correlations }\end{array}$ \\
\hline 8 & $136.7\left(\mathrm{~J}_{\mathrm{C}-\mathrm{H}}=217.8\right)$ & 8.81 (bs) & C 1' \\
\hline $1^{\prime}$ & $89.95\left(\mathrm{~J}_{\mathrm{C}-\mathrm{H}}=172.5\right)$ & $6.05\left(\mathrm{~J}_{1^{\prime}-2^{\prime}}=4.1\right)$ & $\mathrm{C} 8$ \\
\hline $2 '$ & $74.60\left(\mathrm{~J}_{\mathrm{C}-\mathrm{H}}=153.5\right)$ & $4.76\left(\mathrm{~J}_{2^{\prime}-3^{\prime}}=5.5\right)$ & \\
\hline $3^{\prime}$ & $70.14\left(\mathrm{~J}_{\mathrm{C}-\mathrm{H}}=152.0\right)$ & $4.49\left(\mathrm{~J}_{3^{\prime}-4},=4.7\right)$ & \\
\hline $4 '$ & $83.54\left(\mathrm{~J}_{\mathrm{C}-\mathrm{H}}=152.0\right)$ & $\begin{aligned} 4.45\left(\mathrm{~J}_{4^{\prime}-5^{\prime} \mathrm{a}}=3.4\right) \\
\left(\mathrm{J}_{4^{\prime}-5^{\prime} \mathrm{b}}=2.6\right)\end{aligned}$ & \\
\hline $\begin{array}{l}\text { 'a } \\
5 \text { 'b }\end{array}$ & $67.32\left(\mathrm{~J}_{\mathrm{C}-\mathrm{H}}=151.3\right)$ & $\begin{array}{l}4.32\left(\mathrm{~J}_{5}{ }^{\mathrm{a}-5^{\prime} \mathrm{b}}=11.6\right) \\
4.41\left(\mathrm{~J}_{5^{\prime} \mathrm{a}-5^{\prime} \mathrm{b}}=11.6\right)\end{array}$ & \\
\hline
\end{tabular}

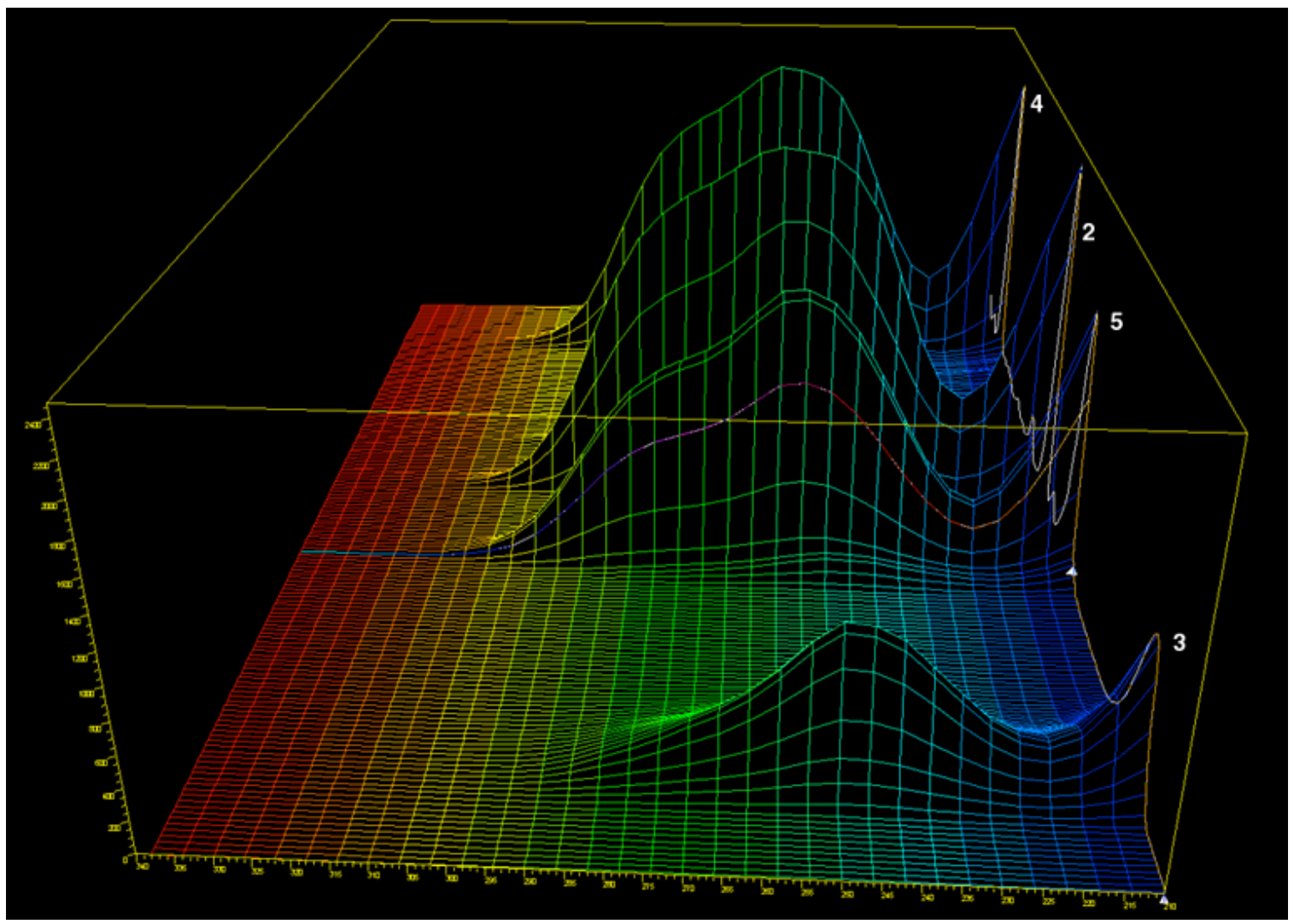

Figure 1. An HPLC chromatogram showing the UV/Vis spectra for $\mathbf{2 - 5}$. 


\section{Partially Characterized Compounds:}

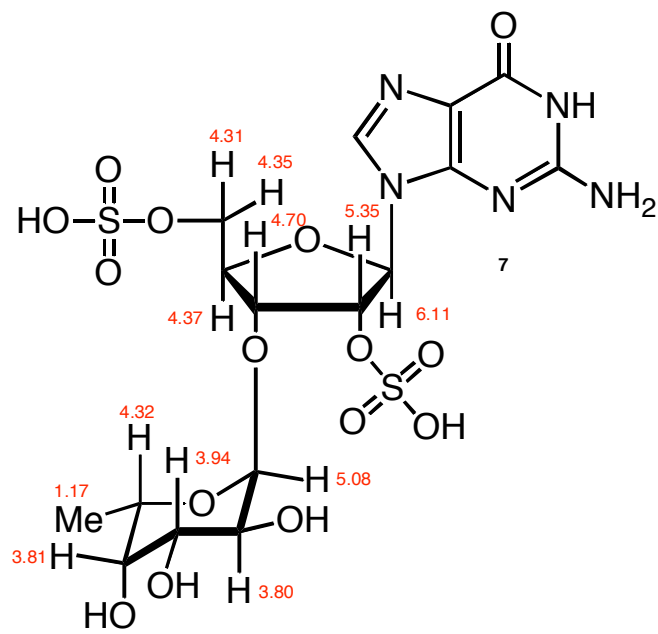

$((2 R, 3 R, 4 S, 5 R)-5$-(2-amino-6-oxo-1,6-dihydropurin-9-yl)-4-hydrogen sulfate-3-((2S,4S,5S)3,4,5-trihydroxy-6-methyl-tetrahydro-2H-pyran-2-yloxy)-tetrahydrofuran-2-yl)methyl hydrogen sulfate (7). ESI-MS (negative ion): (M-1)/Z = 588.06 AMU, (M-2)/Z = 293.53 AMU.

\begin{tabular}{|c|c|}
\hline Position & $\delta{ }^{1} \mathbf{H}[\mathrm{ppm}]$ \\
\hline $1^{\prime}$ & $6.11\left(\mathrm{~J}_{1^{\prime}-2},=5.0\right)$ \\
\hline 2 & $5.35\left(\mathrm{~J}_{2^{\prime}-3},=5.5\right)$ \\
\hline 3, & $4.70\left(\mathrm{~J}_{3^{\prime}-4},=3.6\right)$ \\
\hline $4{ }^{\prime}$ & $\begin{aligned} 4.37\left(\mathrm{~J}_{4^{\prime}-5}{ }^{\prime} \mathrm{a}\right. & =3.2) \\
\left(\mathrm{J}_{4^{\prime}-5{ }^{\prime} \mathrm{b}}\right. & =2.6)\end{aligned}$ \\
\hline $\begin{array}{l}\text { 5'a } \\
\text { 5'b }\end{array}$ & 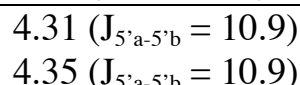 \\
\hline 1, & $5.08\left(\mathrm{~J}_{1^{\prime},-2,}=4.3\right)$ \\
\hline 2, & $3.80\left(\mathrm{~J}_{2,3^{\prime},}=10.6\right)$ \\
\hline 3"' & $3.94\left(\mathrm{~J}_{3,{ }^{\prime \prime}-4,}=3.4\right)$ \\
\hline $4 "$ & $3.81\left(\mathrm{~J}_{4,-5,},<1\right)$ \\
\hline 5, & $4.32\left(\mathrm{~J}_{5^{\prime \prime}-6,}=6.5\right)$ \\
\hline $6 "$ & 1.17 \\
\hline
\end{tabular}




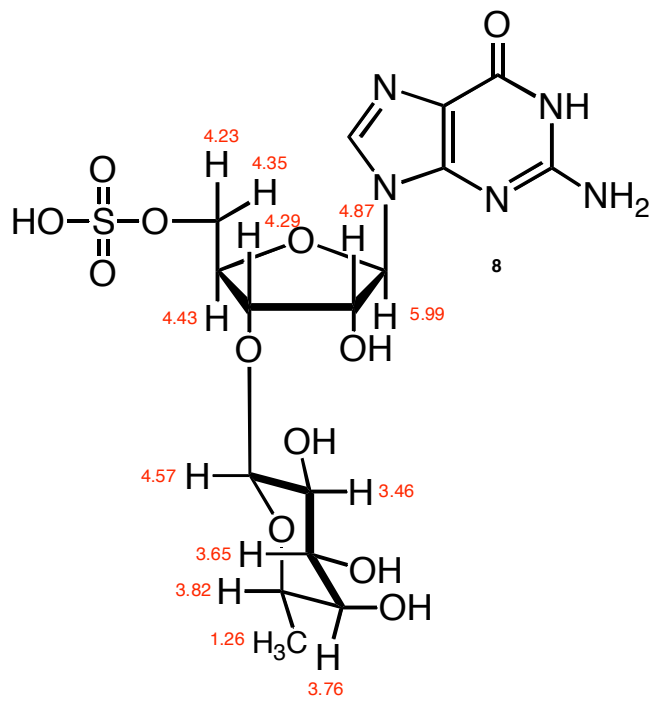

$((2 R, 3 R, 4 S, 5 R)-5$-(2-amino-6-oxo-1,6-dihydropurin-9-yl)-4-hydrogen sulfate-3-( $(2 R, 4 S, 5 S)$ 3,4,5-trihydroxy-6-methyl-tetrahydro-2H-pyran-2-yloxy)-tetrahydrofuran-2-yl)methyl hydrogen sulfate (8). ESI-MS (negative ion): (M-1)/Z = 508.10 AMU.

\begin{tabular}{|c|c|}
\hline Position & $\delta{ }^{1} H[p p m]$ \\
\hline $1^{\prime}$ & $5.99\left(\mathrm{~J}_{1^{\prime}-2},=4.0\right)$ \\
\hline 2 ' & $4.87\left(\mathrm{~J}_{2^{\prime}-3},=6.2\right)$ \\
\hline $3^{\prime}$ & $4.67\left(\mathrm{~J}_{3^{\prime}-4^{\prime}}=4.0\right)$ \\
\hline $4{ }^{\prime}$ & $\begin{aligned} & .43\left(\mathrm{~J}_{4^{\prime}-5}{ }^{\prime} \mathrm{a}\right.=4.8) \\
&\left(\mathrm{J}{ }^{\prime}-5{ }^{\prime} \mathrm{b}\right.=3.3) \\
&\end{aligned}$ \\
\hline 5 'a & $4.23\left(\mathrm{~J}_{5}{ }^{\prime} \mathrm{a}-5^{\prime} \mathrm{b}=11.4\right)$ \\
\hline 5 'b & $4.29\left(\mathrm{~J}_{5}{ }^{\prime} \mathrm{a}-5^{\prime} \mathrm{b}=11.4\right)$ \\
\hline $1, x$ & $4.57\left(\mathrm{~J}_{1,{ }^{\prime}-2,}=8.3\right)$ \\
\hline 2, & $3.46\left(\mathrm{~J}_{2^{\prime \prime}-3^{\prime \prime}}=10.4\right)$ \\
\hline 3, & $3.65\left(\mathrm{~J}_{3,-4,}=3.5\right)$ \\
\hline $4 "$ & $3.76\left(\mathrm{~J}_{4,},-5,<1\right)$ \\
\hline 5, & $3.82\left(\mathrm{~J}_{5},-6,=6.9\right)$ \\
\hline $6 "$ & 1.26 \\
\hline
\end{tabular}




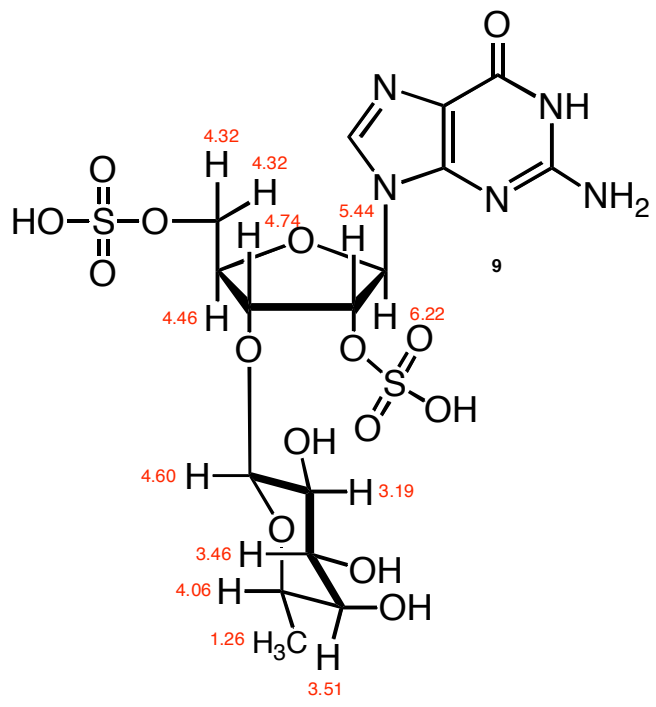

$((2 R, 3 R, 4 S, 5 R)-5$-(2-amino-6-oxo-1,6-dihydropurin-9-yl)-4-hydrogen sulfate-3-( $(2 R, 4 S, 5 S)$ 3,4,5-trihydroxy-6-methyl-tetrahydro-2 $\mathrm{H}$-pyran-2-yloxy)-tetrahydrofuran-2-yl)methyl hydrogen sulfate (9). ESI-MS (negative ion): (M-1)/Z = 588.06 AMU, (M-2)/Z = 293.53 AMU.

\begin{tabular}{|c|c|}
\hline Position & $\delta^{1} \mathbf{H}[\mathrm{ppm}]$ \\
\hline $1^{\prime}$ & $6.22\left(\mathrm{~J}_{1^{\prime}-2},=5\right)$ \\
\hline $2^{\prime}$ & $5.44\left(\mathrm{~J}_{2^{\prime}-3^{\prime}}=5.5\right)$ \\
\hline $3^{\prime}$ & $4.74\left(\mathrm{~J}_{3^{\prime}-4}=3.6\right)$ \\
\hline $4{ }^{\prime}$ & $\begin{aligned} 4.46\left(\mathrm{~J}_{4^{\prime}-5^{\prime} \mathrm{a}}=4.0\right) \\
\left(\mathrm{J}^{\prime} 5^{\prime} \mathrm{b}=3.2\right)\end{aligned}$ \\
\hline 5'a & $4.32\left(\mathrm{~J}_{5}{ }^{\prime} \mathrm{a}-5^{\prime} \mathrm{b}=11.3\right)$ \\
\hline $5^{\prime} \mathrm{b}$ & $4.32\left(\mathrm{~J}_{5{ }^{\prime} \mathrm{a}-5^{\prime} \mathrm{b}}=11.3\right)$ \\
\hline $1 "$, & $4.60\left(\mathrm{~J}_{1{ }^{\prime \prime}-2,}=7.9\right)$ \\
\hline $2 "$ & $3.19\left(\mathrm{~J}_{2,{ }^{\prime}-3,}=9.9\right)$ \\
\hline $3 "$ & $3.46\left(\mathrm{~J}_{3^{\prime \prime}-4,}=3.3\right)$ \\
\hline 4"' & $3.51\left(\mathrm{~J}_{4,-5},<1\right)$ \\
\hline $5 "$ & $4.06\left(\mathrm{~J}_{5^{\prime \prime}-6,},=6.8\right)$ \\
\hline $6 "$ & 1.26 \\
\hline
\end{tabular}

